\title{
Optimum-Point Formulas for Osculatory and Hyperosculatory Interpolation
}

\author{
By Herbert E. Salzer
}

\begin{abstract}
Formulas are given for $n$-point osculatory and hyperosculatory (as well as ordinary) polynomial interpolation for $f(x)$, over $(-1,1)$, in terms of $f\left(x_{i}\right), f^{\prime}\left(x_{i}\right)$ and $f^{\prime \prime}\left(x_{i}\right)$ at the irregularly-spaced Chebyshev points $x_{i}=-\cos$ $\{(2 i-1) \pi / 2 n\}, i=1, \cdots, n$. The advantage over corresponding formulas for $x_{i}$ equally spaced is in the squaring and cubing, in the respective osculatory and hyperosculatory formulas, of the approximate ratio of upper bounds for the remainder in ordinary interpolation using Chebyshev and equal spacing (e.g., for $n=10$, the 15 per cent ratio for ordinary interpolation becoming 2.4 per cent and 0.37 per cent for osculatory and hyperosculatory interpolation). The upper bounds for the remainders in these optimum $n$-point $r$-ply confluent formulas (here $r=1$ and 2) are around $2^{r}$ times those of the optimum $\{(r+1) n\}$-point non-confluent formulas. But these present confluent formulas may require fewer computations for irregular arguments when $f(x)$ satisfies a simple first or second-order differential equation. To facilitate computation, for $n=2(1) 10$, auxiliary quantities $a_{i}, b_{i}$ and $c_{i}, i=1, \cdots, n$, independent of $x$, are tabulated exactly or to $15 \mathrm{~S}$, not precisely for the optimum points, but for those Chebyshev arguments rounded to $2 \mathrm{D}$ ("near-optimum" points). At the very worst ( $n=9$, hyperosculatory) this change about doubles the remainder, which is still less than $\left(\frac{1}{50}\right)$ th of the remainder in the corresponding equally-spaced formula.
\end{abstract}

1. Advantage Over Equal-Interval Formulas. Formulas are given here for $n$-point osculatory and hyperosculatory polynomial interpolation for $f(x)$, from prescribed values of $f(x)$ with its first, or first and second derivatives at the irregularly-spaced Chebyshev points $x_{n-i+1}=\cos \{(2 i-1) \pi / 2 n\}, i=1,2, \cdots, n$, instead of equally-spaced points. In this notation, $x_{i}=-x_{n-i+1}$ and $x_{i}$ increases with $i$. For the sake of completeness, the ordinary Lagrangian interpolation formulas are also given for these Chebyshev points. All $n$-point ordinary, osculatory and hyperosculatory formulas given here are exact for $f(x)$ a polynomial of degree $n-1,2 n-1$ and $3 n-1$ respectively.

The advantage of Chebyshev-point over equal-interval polynomial interpolation formulas is apparent from the factor $\Pi(x) \equiv \Pi_{i=1}^{n}\left(x-x_{i}\right)$ in the remainder term, which is $\Pi(x) f^{(n)}(\xi) / n$ ! for $n$-point ordinary Lagrangian interpolation, $\{\Pi(x)\}^{2} f^{(2 n)}(\xi) /(2 n)$ ! for $n$-point osculatory interpolation and $\{\Pi(x)\}^{3} f^{(3 n)}(\xi) /(3 n)$ ! for $n$-point hyperosculatory interpolation. At the moment, in order to compare Chebyshev-point with equal-interval formulas, let the range of $x$ be $(-1,1)$, since the relative improvement of the former over the latter is unchanged under any linear transformation. For $x_{i}$ at the Chebyshev points, $|\Pi(x)| \leqq\left(\frac{1}{2}\right)^{n-1}$, which is a fraction of the upper bound of $|\Pi(x)|$ for equally-spaced $x_{i}$ 's. However, that fraction is not impressively small, decreasing rather slowly with increasing $n$ (except

Received August 28, 1961. 
Schedule 1: Upper Bound for Absolute Value of Coefficient of $f^{(m)}(\xi)$

\begin{tabular}{|c|c|c|c|c|c|c|}
\hline \multirow[t]{2}{*}{$n$} & \multicolumn{2}{|c|}{ Ordinary: $m=n$} & \multicolumn{2}{|c|}{ Osculatory: $m=2 n$} & \multicolumn{2}{|c|}{$\begin{array}{l}\text { Hyperosculatory: } \\
m=3 n\end{array}$} \\
\hline & $U . B$. & $\begin{array}{l}\text { Ratio to } U . B . \\
\text { for equal } \\
\text { spacing }\end{array}$ & $U . B$. & $\begin{array}{l}\text { Ratio to } U . B . \\
\text { for equal } \\
\text { spacing }\end{array}$ & $U . B$. & $\begin{array}{l}\text { Ratio to } U . B . \\
\text { for equal } \\
\text { spacing }\end{array}$ \\
\hline $\begin{array}{r}2 \\
3 \\
4 \\
5 \\
6 \\
7 \\
8 \\
9 \\
10\end{array}$ & $\begin{array}{l}.250 \\
.(1) 417 \\
.(2) 521 \\
.(3) 521 \\
.(4) 434 \\
.(5) 310 \\
.(6) 194 \\
.(7) 108 \\
.(9) 538\end{array}$ & $\begin{array}{l}50 \% \\
65 \% \\
63 \% \\
55 \% \\
45 \% \\
36 \% \\
27 \% \\
21 \% \\
15 \%\end{array}$ & $\begin{array}{l}.(1) 104 \\
.(4) 868 \\
.(6) 388 \\
.(8) 108 \\
.(11) 204 \\
.(14) 280 \\
.(17) 292 \\
.(20) 238 \\
.(23) 157\end{array}$ & $\begin{array}{l}25 \% \\
42 \% \\
40 \% \\
30 \% \\
20 \% \\
13 \% \\
7.6 \% \\
4.3 \% \\
2.4 \%\end{array}$ & $\begin{array}{l}.(3) 174 \\
.(7) 431 \\
.(11) 408 \\
.(15) 187 \\
.(20) 477 \\
.(25) 747 \\
.(30) 769 \\
.(35) 547 \\
.(40) 281\end{array}$ & $\begin{array}{l}12 \frac{1}{2} \% \\
27 \% \\
25 \% \\
17 \% \\
9.2 \% \\
4.5 \% \\
2.1 \% \\
0.90 \% \\
0.37 \%\end{array}$ \\
\hline
\end{tabular}

for a slight increase from $n=2$ to $n=3$ ) being somewhat larger than $\frac{1}{9}$ for $n=11$. Thus ordinary Lagrangian interpolation at Chebyshev points, even for $n=9$ or 10, gains less than one full decimal place accuracy over interpolation at equallyspaced points. But in the osculatory and hyperosculatory cases, the $\{\Pi(x)\}^{2}$ and $\{\Pi(x)\}^{3}$ in the remainder term squares and cubes the relative improvement of the Chebyshev-point formulas. For instance, when $n=10$ the approximately 15 per cent ratio in the upper bounds of $|\Pi(x)|$ for the Chebyshev and equally-spaced points is now replaced by only around 2 per cent and 0.4 per cent in the ratios of the upper bounds of $\{\Pi(x)\}^{2}$ and $\left|\{\Pi(x)\}^{3}\right|$ respectively.

In Schedule 1, we give the upper bound for the absolute value of the coefficient of $f^{(m)}(\xi),-1 \leqq \xi \leqq 1, m=n, 2 n$ and $3 n$, in the remainder term of the $n$-point ordinary, osculatory and hyperosculatory interpolation formulas, for $n=2(1) 10$ to $3 \mathrm{~S}$. These bounds are, of course, $1 / 2^{n-1} n$ !, $1 / 2^{2 n-2}(2 n)$ ! and $1 / 2^{3 n-3}(3 n)$ ! respectively. Next to each upper bound is the ratio, in per cent, of that quantity to the corresponding upper bound when the $n$ points $x_{i}$ are equally-spaced over $(-1,1)$. The quantity in parentheses indicates the number of zeros between the decimal point and the first significant digit.

2. Comparison with Non-Osculatory Chebyshev-Point Formulas. The upper bounds for $\{\Pi(x)\}^{2}$ and $\left|\{\Pi(x)\}^{3}\right|$ in the $n$-point Chebyshev osculatory and hyperosculatory formulas are $\left(\frac{1}{2}\right)^{2 n-2}$ and $\left(\frac{1}{2}\right)^{3 n-3}$ respectively, which is only twice and four times the upper bounds of $\left(\frac{1}{2}\right)^{2 n-1}$ and $\left(\frac{1}{2}\right)^{3 n-1}$ for $|\Pi(x)|$ in the $2 n$ - and $3 n$-point optimum-point (non-confluent) formulas of the same degree of accuracy, namely, for $x_{i}$ at the zeros of the Chebyshev polynomials $T_{2 n}(x)=\left(\frac{1}{2}\right)^{2 n-1} \cos \left(2 n \cos ^{-1} x\right)$ and $T_{3 n}(x)=\left(\frac{1}{2}\right)^{3 n-1} \cos \left(3 n \cos ^{-1} x\right)$. This two-and four-ratio is unchanged, of course, under a linear transformation to any range $(a, b)$ other than $(-1,1)$, because the factor of $\{(b-a) / 2\}^{2 n}$ or $\{(b-a) / 2\}^{3 n}$ which then enters the remainder term is the same for both confluent and non-confluent forms of the interpolation formulas.

The confluent Chebyshev-point formulas given here, while not quite as ac- 
curate as the non-confluent Chebyshev-point formulas of the same degree, have this advantage: For irregularly-spaced values of $x_{i}$, it is often less work to compute $n$ values of $y_{i} \equiv f\left(x_{i}\right)$ together with $y_{i}^{\prime} \equiv f^{\prime}\left(x_{i}\right)$, or with $y_{i}^{\prime}$ and $y_{i}^{\prime \prime} \equiv f^{\prime \prime}\left(x_{i}\right)$, instead of $2 n$ or $3 n$ values of $y_{i}$. For instance, in the osculatory case $y=f(x)$ might satisfy a rather simple first-order differential equation $y^{\prime}=\phi(x, y)$ where it is easier to obtain $n$ values of $y_{i}^{\prime}=\phi\left(x_{i}, y_{i}\right)$ after $y_{i}$ has been calculated than to compute $n$ more values of $y_{i}$. The most obvious example is when $\phi(x, y)=y$, where $y=e^{x}$ and obtaining $y_{i}{ }^{\prime}=y_{i}$ involves no extra work at all. In the hyperosculatory case $y$ might satisfy a simple second-order differential equation from which $y_{i}{ }^{\prime \prime}$ is readily obtained from $y_{i}$ and $y_{i}{ }^{\prime}$.

3. Interpolation Formulas. We shall not repeat here the derivations of the interpolation formulas, since they have been given a number of times, as well as a full discussion of their advantages, efficient arrangement, remainder terms, extension to inverse and complex interpolation, etc., in previous articles [1]-[3]. In (1)-(14) below, $n$ is understood, $i$ ranges from 1 to $n, f \equiv f(x), f_{i} \equiv f\left(x_{i}\right)$, $f_{i}^{\prime} \equiv f^{\prime}\left(x_{i}\right), f_{i}^{\prime \prime} \equiv f^{\prime \prime}\left(x_{i}\right)$ and $\sum$ denotes $\sum_{i=1}^{n}$. We employ quantities $p_{i j}, q_{i}$, $r_{i}$ and $s_{i}$ given by

$$
\left\{\begin{array}{l}
p_{i j}=1 /\left(x_{i}-x_{j}\right), j \neq i ; \quad q_{i}=\sum_{j=1, j \neq i}^{n} p_{i j} ; \\
r_{i}=q_{i}{ }^{2} ; \quad s_{i}=\sum_{j=1, j \neq i}^{n} p_{i j}^{2} .
\end{array}\right.
$$

For each $n$ we define first

$$
A_{i}=\prod_{j=1, j \neq i}^{n} p_{i j}
$$

For ordinary interpolation we define

$$
a_{i}=k_{1}(n) A_{i}
$$

For osculatory interpolation we define

$$
\left\{\begin{array}{l}
a_{i}=k_{2}(n) A_{i}{ }^{2}, \\
b_{i}=-2 q_{i} a_{i}=-2 k_{2}(n) q_{i} A_{i}{ }^{2} .
\end{array}\right.
$$

For hyperosculatory interpolation we define

$$
\left\{\begin{array}{l}
a_{i}=k_{3}(n) A_{i}{ }^{3}, \\
b_{i}=-3 q_{i} a_{i}=-3 k_{3}(n) q_{i} A_{i}{ }^{3}, \\
c_{i}=a_{i}\left[\frac{9}{2} r_{i}+\frac{3}{2} s_{i}\right]=k_{3}(n)\left[\frac{9}{2} r_{i}+\frac{3}{2} s_{i}\right] A_{i}{ }^{3} .
\end{array}\right.
$$

In $(3)-(5)$, the $k_{m}(n), m=1,2,3$, denote suitably chosen constants that do not affect the results of the interpolation in formulas (7), (10) and (14), but which might (and this depends upon the values and functional nature of the arguments $x_{i}$ ) facilitate appreciably the calculation and use of the auxiliary quantities $a_{i}$, $a_{i}$ and $b_{i}$, or $a_{i}, b_{i}$ and $c_{i}$ in (6)-(14).

For ordinary $n$-point interpolation, of $(n-1)$ th degree accuracy, we obtain

$$
\begin{gathered}
\alpha_{i}=a_{i} /\left(x-x_{i}\right), \text { from which } \\
f \sim \Sigma \alpha_{i} f_{i} / \Sigma \alpha_{i} .
\end{gathered}
$$


For $n$-point polynomial osculatory interpolation of $(2 n-1)$ th degree accuracy, we obtain

$$
\begin{aligned}
\beta_{i} & =a_{i} /\left(x-x_{i}\right), \\
\alpha_{i} & =\left(\beta_{i}+b_{i}\right) /\left(x-x_{i}\right), \quad \text { from which } \\
f & \sim \Sigma\left(\alpha_{i} f_{i}+\beta_{i} f_{i}{ }^{\prime}\right) / \Sigma \alpha_{i} .
\end{aligned}
$$

For $n$-point polynomial hyperosculatory interpolation of $(3 n-1)$ th degree accuracy, we obtain

$$
\begin{aligned}
\gamma_{i} & =a_{i} / 2\left(x-x_{i}\right), \\
\beta_{i} & =\left(2 \gamma_{i}+b_{i}\right) /\left(x-x_{i}\right), \\
\alpha_{i} & =\left(\beta_{i}+c_{i}\right) /\left(x-x_{i}\right), \text { from which } \\
f & \sim \Sigma\left(\alpha_{i} f_{i}+\beta_{i} f_{i}^{\prime}+\gamma_{i} f_{i}^{\prime \prime}\right) / \Sigma \alpha_{i} .
\end{aligned}
$$

4. Use of "Near-Optimum" Points. Instead of taking the $x_{i}$ precisely equal to the zeros of $T_{n}(x)$, we now round them off to two decimal places. This makes the osculatory and hyperosculatory formulas "near-optimum" rather than "optimum" point formulas. Three reasons for such a choice are: 1) easier calculation and checking of the table of the auxiliary quantities $a_{i}, b_{i}$ and $c_{i}$ occurring in the interpolation formulas (7), (10) and $(14) ; 2$ ) some of the $a_{i}$, for the lower values of $n$, can be given exactly with much fewer than 15 significant figures; 3) for many functions $f(x)$, it is less work to calculate $f\left(x_{i}\right)$ when $x_{i}$ is an exact two-decimal argument.

The employment of rounded-off zeros of $T_{n}(x)$ as the arguments $x_{i}$ was suggested by Lanczos's use of rounded zeros of Legendre polynomials for a modification of Gaussian quadrature. [4] In this present case, the slight shift in the $x_{i}$ from exact to rounded Chebyshev points does not produce too great a change in the upper bound for the remainder, (the changes for $n=7$ and $n=9$ being appreciably greater than the rest, as seen in Schedule 2). This justifies the terminology "near-optimum", which contrasts sharply with the experience of Lanczos with rounded Gaussian points for quadrature formulas. Thus, quoting his comment on an example [4, p. 410]: "Compared with the Gaussian error, the error has increased by the factor 71, which shows the great sensitivity of the Gaussian method to even small shifts of the zeros." Here, at the worst, for 9-point hyperosculatory interpolation, the choice of the near-optimum instead of optimum points causes the maximum error to be slightly more than doubled. But even then it is less than $\left(\frac{1}{30}\right)$ th of the maximum error in the corresponding equally-spaced formula.

In attempting to estimate the sensitivity in the upper bound of the absolute value of $\Pi(x)=T_{n}(x)$ for a slight change of $\Delta x_{i}$ in every $x_{i}$, we differentiate $T_{n}(x)=\Pi_{i=1}^{n}\left(x-x_{i}\right)$ partially with respect to each $x_{i}$, obtaining for $D_{n}(x)$, the dominant part of the deviation in $\Pi(x)$, the expression

$$
D_{n}(x)=-\sum_{i=1}^{n} \frac{\Pi_{i=1}^{n}\left(x-x_{i}\right)}{x-x_{i}} \Delta x_{i}
$$


TABLES of $a_{i}, b_{i}$ and $c_{i}$

\begin{tabular}{|c|c|c|c|c|c|}
\hline \multirow[b]{2}{*}{$n$} & \multirow[b]{2}{*}{$i$} & \multirow[b]{2}{*}{$x_{i}$} & \multirow{2}{*}{$\frac{\text { Ordinary Interpolation }}{a_{i}}$} & \multicolumn{2}{|c|}{ Osculatory Interpolation } \\
\hline & & & & $a_{i}$ & $b_{i}$ \\
\hline 2 & 1,2 & $\mp 0.71$ & $\mp 1$ & 1 & \pm 1.40845070422535 \\
\hline $\begin{array}{l}3 \\
3\end{array}$ & $\frac{1}{2}, 3$ & $\left|\begin{array}{c}\mp 0.87 \\
0\end{array}\right|$ & $\begin{array}{r}1 \\
-2\end{array}$ & $\begin{array}{l}1 \\
4\end{array}$ & $\pm 3.44827 \underset{0}{58620} 6897$ \\
\hline $\begin{array}{l}4 \\
4 \\
\end{array}$ & $\begin{array}{ll}1,4 \\
2,3\end{array}$ & $\begin{array}{l}\mp 0.92 \\
\mp 0.38\end{array}$ & $\begin{array}{l}\mp 1.9 \\
\pm 4.6\end{array}$ & $\begin{array}{l}0.361 \\
2.116\end{array}$ & $\begin{array}{lll} \pm 2.28481 & 29567 & 6948 \\
\pm 0.98676 & 86309 & 79157\end{array}$ \\
\hline $\begin{array}{l}5 \\
5 \\
5\end{array}$ & $\begin{array}{l}1,5 \\
2, \\
3\end{array}$ & $\left|\begin{array}{c}\mp 0.95 \\
\mp 0.59 \\
0\end{array}\right|$ & $\begin{array}{r}0.3481 \\
-0.9025 \\
1.1088\end{array}$ & $\begin{array}{l}0.12117361 \\
0.81450625 \\
1.22943 \quad 744\end{array}$ & \begin{tabular}{|ccc} 
\pm 1.21320 & 85521 & 6070 \\
\pm 0.67432 & 28058 & 42933 \\
0 & 0
\end{tabular} \\
\hline $\begin{array}{l}6 \\
6 \\
6\end{array}$ & $\begin{array}{ll}1, & 6 \\
2, & 5 \\
3, & 4\end{array}$ & $\begin{array}{l}\mp 0.97 \\
\mp 0.71 \\
\mp 0.26\end{array}$ & $\begin{array}{l}\mp 1.5 \\
\pm 4.1 \\
\mp 5.6\end{array}$ & $\begin{array}{l}0.225 \\
1.681 \\
3.136\end{array}$ & \begin{tabular}{|lll} 
\pm 3.23024 & 16119 & 5097 \\
\pm 2.37511 & 73717 & 7847 \\
\pm 0.85512 & 42401 & 72493
\end{tabular} \\
\hline $\begin{array}{l}7 \\
7 \\
7 \\
7\end{array}$ & $\begin{array}{l}1,7 \\
2,6 \\
3,5 \\
4\end{array}$ & $\mid \begin{array}{c}\mp 0.97 \\
\mp 0.78 \\
\mp 0.43 \\
0\end{array}$ & $\begin{array}{r}0.37810201 \\
-1.04383446 \\
1.51061495 \\
-1.68976500\end{array}$ & $\begin{array}{lll}0.14296 & 11299 & 66040 \\
1.08959 & 03798 & 8349 \\
2.28195 & 75271 & 6350 \\
2.85530 & 57552 & 2500\end{array}$ & \begin{tabular}{|ccc} 
\pm 2.84410 & 18250 & 1904 \\
\pm 1.99381 & 25356 & 4272 \\
\pm 1.46095 & 20152 & 4591 \\
0 & \multicolumn{3}{c}{0}
\end{tabular} \\
\hline $\begin{array}{l}8 \\
8 \\
8 \\
8\end{array}$ & $\begin{array}{ll}1,8 \\
2,7 \\
3,6 \\
4,5\end{array}$ & $\mid \begin{array}{l}\mp 0.98 \\
\mp 0.83 \\
\mp 0.56 \\
\mp 0.20\end{array}$ & $\begin{array}{l}\mp 0.9753656688 \\
\pm 2.81517 \quad 71648 \\
\mp 4.1539120805 \\
\pm 4.7272603686\end{array}$ & 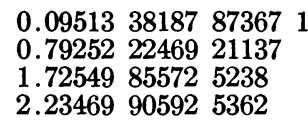 & $\begin{array}{lll} \pm 2.45239 & 17122 & 6555 \\
\pm 2.32927 & 47108 & 1650 \\
\pm 0.93364 & 45598 & 54158 \\
\mp 0.05814 & 57965 & 08793\end{array}$ \\
\hline $\begin{array}{l}9 \\
9 \\
9 \\
9 \\
9\end{array}$ & $\begin{array}{l}1,9 \\
2,8 \\
3,7 \\
4,6 \\
5\end{array}$ & $\begin{array}{c}\mp 0.98 \\
\mp 0.87 \\
\mp 0.64 \\
\mp 0.34 \\
0\end{array}$ & 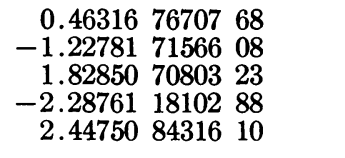 & $\begin{array}{lll}\mathbf{0 . 2 1 4 5 2} & 42912 & 44654 \\
1.50753 & 49700 & 6095 \\
3.34343 & 81427 & 9134 \\
\mathbf{5} .23316 & 77945 & 6914 \\
\mathbf{5 . 9 9 0 2 9} & 75228 & 0204\end{array}$ & 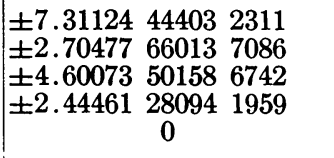 \\
\hline $\begin{array}{l}10 \\
10 \\
10 \\
10 \\
10\end{array}$ & $\begin{array}{l}1,10 \\
2,9 \\
3,8 \\
4,7 \\
5,6\end{array}$ & $\begin{array}{l}\mp 0.99 \\
\mp 0.89 \\
\mp 0.71 \\
\mp 0.45 \\
\mp 0.16\end{array}$ & 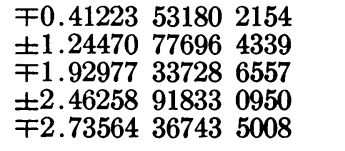 & $\begin{array}{lll}0.16993 & 79574 & 24320 \\
1.54929 & 74318 & 1062 \\
3.72402 & 52706 & 2096 \\
6.06434 & 54857 & 5295 \\
7.48374 & 63130 & 1161\end{array}$ & $\begin{array}{|lll|} \pm 6.73542 & 63847 & 8561 \\
\pm 8.10439 & 99566 & 8827 \\
\pm 3.47307 & 39064 & 2641 \\
\pm 6.43767 & 06135 & 5020 \\
\mp 1.57793 & 67089 & 3871\end{array}$ \\
\hline
\end{tabular}

Hyperosculatory Interpolation

\begin{tabular}{|c|c|c|c|c|c|c|}
\hline$n$ & $i$ & $x_{i}$ & $a_{i}$ & & $b_{i}$ & $c_{i}$ \\
\hline 2 & 1,2 & $\mp 0.71$ & $\mp 1$ & -2.11267 & 605633803 & $\mp 2.9756000793 \quad 4934$ \\
\hline $\begin{array}{l}3 \\
3\end{array}$ & $\frac{1}{2}, 3$ & $\underset{0}{\mp 0.87}$ & $\begin{array}{r}1 \\
-8\end{array}$ & \pm 5.17241 & $\begin{array}{l}379310345 \\
0\end{array}$ & $\begin{array}{r}15.8541418945700 \\
-31.7082837891399\end{array}$ \\
\hline $\begin{array}{l}4 \\
4\end{array}$ & $\begin{array}{l}1,4 \\
2,3\end{array}$ & $\begin{array}{l}\mp 0.92 \\
\mp 0.38\end{array}$ & $\begin{array}{l}\mp 0.6859 \\
\pm 9.7336\end{array}$ & $\begin{array}{r}-6.51171 \\
6.80870\end{array}$ & $\begin{array}{l}692679301 \\
355375619\end{array}$ & $\begin{array}{l}\mp 35.3510561593 \quad 570 \\
\pm 86.36831 \quad 12988 \quad 727\end{array}$ \\
\hline $\begin{array}{l}5 \\
5 \\
5\end{array}$ & $\begin{array}{ll}1,5 \\
2,4 \\
3\end{array}$ & $\begin{array}{l}\mp 0.95 \\
\mp 0.59 \\
0\end{array}$ & $\begin{array}{rrr}0.04218 & 05336 & 41 \\
-0.73509 & 18906 & 25 \\
1.36320 & 02334 & 72\end{array}$ & $\begin{array}{l} \pm 0.63347 \\
\mp 0.91286\end{array}$ & $\begin{array}{ll}68455 & 10712 \\
44984 & 09870 \\
0 & \end{array}$ & $\begin{array}{r}5.35936149295057 \\
-13.4992433461453 \\
16.2797637063895\end{array}$ \\
\hline $\begin{array}{l}6 \\
6 \\
6\end{array}$ & $\begin{array}{l}1,6 \\
2,5 \\
3,4\end{array}$ & $\begin{array}{l}\mp 0.97 \\
\mp 0.71 \\
\mp 0.26\end{array}$ & $\begin{array}{l}\mp 0.03375 \\
\pm 0.68921 \\
\mp 1.75616\end{array}$ & $\begin{array}{r}-0.72680 \\
1.46069 \\
-0.71830\end{array}$ & $\begin{array}{l}4362688967 \\
718364376 \\
4361744894\end{array}$ & $\begin{array}{rll}\mp 8.7400129929 & 3812 \\
\pm 23.92400 & 98678 & 306 \\
\mp 32.66402 & 28607 & 687\end{array}$ \\
\hline $\begin{array}{l}7 \\
7 \\
7 \\
7\end{array}$ & $\begin{array}{l}1,7 \\
2,6 \\
3,5 \\
4\end{array}$ & $\begin{array}{c}\mp 0.97 \\
\mp 0.78 \\
\mp 0.43 \\
0\end{array}$ & $\begin{array}{rlll}0.00540 & 53890 & 59203 & 10 \\
-0.11373 & 51985 & 80688 \\
0.34471 & 59155 & 79822 \\
-0.48247 & 95729 & 47777\end{array}$ & $\begin{array}{l} \pm 0.16130 \\
\mp 0.31218 \\
\pm 0.33104\end{array}$ & $\begin{array}{l}4092502655 \\
1534722577 \\
03933 \quad 19465 \\
0\end{array}$ & $\begin{array}{rll}2.67672 & 79073 & 0026 \\
-7.06970 & 10338 & 4325 \\
10.26580 & 84638 & 704 \\
-11.74567 & 06746 & 548\end{array}$ \\
\hline
\end{tabular}


TABLES of $a_{i}, b_{i}$ and $c_{i}-($ Continued $)$

Hyperosculatory Interpolation

\begin{tabular}{|c|c|c|c|c|c|}
\hline$n$ & $i$ & $x_{i}$ & $a_{i}$ & $b_{i}$ & $c_{i}$ \\
\hline $\begin{array}{l}8 \\
8 \\
8 \\
8\end{array}$ & $\begin{array}{l}1,8 \\
2,7 \\
3,6 \\
4,5\end{array}$ & $\begin{array}{l}\mp 0.98 \\
\mp 0.83 \\
\mp 0.56 \\
\mp 0.20\end{array}$ & $\begin{array}{lll}\mp 0.00927 & 90260 & 78703 \\
\pm 0.22310 & 90532 & 12837 \\
\mp 0.71675 & 69301 & 85600 \\
\pm 1.05640 & 04298 & 5573\end{array}$ & $\begin{array}{rrr}-0.35879 & 68023 & 89020 \\
0.98359 & 81464 & 65512 \\
-0.58174 & 16124 & 10694 \\
-0.04123 & 05479 & 15504\end{array}$ & $\begin{array}{rrr}\mp 7.68100 & 77077 & 0057 \\
\pm 23.18826 & 27433 & 073 \\
\mp 33.10310 & 66120 & 670 \\
\pm 34.10349 & 18155 & 463\end{array}$ \\
\hline $\begin{array}{l}9 \\
9 \\
9 \\
9 \\
9\end{array}$ & $\begin{array}{l}1,9 \\
2,8 \\
3,7 \\
4,6 \\
5\end{array}$ & $\begin{array}{c}\mp 0.98 \\
\mp 0.87 \\
\mp 0.64 \\
\mp 0.34 \\
0\end{array}$ & $\begin{array}{rll}0.00993 & 60716 & 29894 \\
-0.18509 & 77300 & 42737 \\
0.61135 & 00316 & 71595 \\
-1.19714 & 56452 & 0752 \\
1.46613 & 03694 & 9105\end{array}$ & $\begin{array}{ccc} \pm 0.50794 & 98086 & 75992 \\
\mp 0.49814 & 56673 & 93253 \\
\pm 1.26187 & 14826 & 8053 \\
\mp 0.83884 & 87701 & 61438 \\
& 0\end{array}$ & 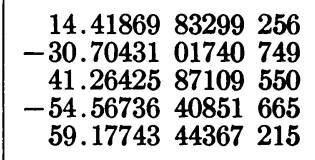 \\
\hline $\begin{array}{l}10 \\
10 \\
10 \\
10 \\
10\end{array}$ & $\begin{array}{l}1,10 \\
2,9 \\
3,8 \\
4,7 \\
5,6\end{array}$ & $\begin{array}{l}\mp 0.99 \\
\mp 0.89 \\
\mp 0.71 \\
\mp 0.45 \\
\mp 0.16\end{array}$ & 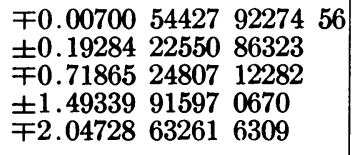 & $\begin{array}{rll}-0.41648 & 70956 & 61415 \\
1.51314 & 14391 & 5812 \\
-1.00533 & 68319 & 9238 \\
2.37800 & 07027 & 9572 \\
0.64750 & 08864 & 49945\end{array}$ & $\begin{array}{rll}\mp 13.63892 & 25685 & 320 \\
\pm 46.53720 & 81301 & 798 \\
\mp 70.78787 & 62690 & 287 \\
\pm 93.6914 & 23293 & 455 \\
\mp 104.39709 & 18091 & 15\end{array}$ \\
\hline
\end{tabular}

so that

$$
\left|D_{n}(x)\right| \leqq 2^{-n+1} \sum_{i=1}^{n} \frac{\left|\Delta x_{i}\right|}{\left|x-x_{i}\right|}
$$

Now for $x$ in the neighborhood of the extrema of $T_{n}(x)$ not close to the ends \pm 1 , the $\left|x-x_{i}\right|$ stays large enough for (16) to furnish upper bounds for $\left|D_{n}(x)\right| / 2^{-n+1}$ of the order of just several per cent when $\Delta x_{i}$ is the roundoff error in employing $x_{i}$ to $2 \mathrm{D}$. However (16) breaks down as a practical formula, for larger $n$ and $x$ either at \pm 1 or at an extremum close to \pm 1 since there $\left|x-x_{i}\right|$ is quite small. This might also be expected from the very large derivative of $2^{n-1} T_{n}(x)$ at $x= \pm 1$, its magnitude being $n^{2}$. Thus, to be on the safe side, to provide for every $x$ in the range $(-1,1)$, instead of using (15) or (16), the factor $\Pi(x)=\Pi_{i=1}^{n}\left(x-x_{i}\right)$ for the chosen near-optimum $x_{i}$ 's was calculated for every $n$ from 2 to 10 , for $x=-1(.001) 1$, and its greatest deviation from zero was found. The percentage increase in the upper bound for the absolute value of the coefficient of $f^{(m)}(\xi)$ (see Schedule 1), due to the use of these near-optimum points $x_{i}$ instead of optimum points, is given in Schedule 2 .

Schedule 2: Increase in Schedule 1 When Using Near-Optimum Points

\begin{tabular}{c|c|c|c}
\hline$n$ & Ordinary & Osculatory & Hyperosculatory \\
\cline { 2 - 3 } 2 & $0.82 \%$ & $1.65 \%$ & $2.5 \%$ \\
3 & $1.4 \%$ & $2.8 \%$ & $4.2 \%$ \\
4 & $5.1 \%$ & $10.5 \%$ & $16 \%$ \\
5 & $1.7 \%$ & $3.4 \%$ & $5.2 \%$ \\
6 & $2.6 \%$ & $5.3 \%$ & $8.0 \%$ \\
7 & $21 \%$ & $46 \%$ & $76 \%$ \\
8 & $6.2 \%$ & $13 \%$ & $20 \%$ \\
9 & $29 \%$ & $66 \%$ & $113 \%$ \\
10 & $7.6 \%$ & $16 \%$ & $25 \%$ \\
\hline
\end{tabular}


5. Tables of Auxiliary Coefficients $a_{i}, b_{i}$ and $c_{i}$. To facilitate the use of (6)-(14) for these near-optimum points $x_{i}$, the auxiliary quantities $a_{i}, b_{i}$ and $c_{i}$ are tabulated here for $n=2(1) 10, i=1, \cdots, n$. It reduced the work considerably to choose the constants $k_{m}(n), m=1,2,3$, in (3)-(5), as products of powers of selected prime numbers $<200$. As a result of this choice, it was easy to give exact values of all the quantities $a_{i}$ for ordinary interpolation, and of $a_{i}$ for $n=2(1) 6$ for osculatory and hyperosculatory interpolation. The remaining quantities $a_{i}$ and all quantities $b_{i}$ and $c_{i}$ are given to $15 \mathrm{~S}$, believed to be correct to within a unit in the last place. In reading entries prefixed by \pm or $\mp$ signs, the upper sign corresponds to the negative $x_{i}$.

General Dynamics/Astronautics San Diego, California

1. H. E. SAlzer, "New formulas for facilitating osculatory interpolation," J. Res. Nat. Bur. Standards, v. 52, 1954, p. 211-216.

2. H. E. SALzen, "Formulae for hyperosculatory interpolation, direct and inverse," Quart. J. Mech. Appl. Math., v. 12, 1959, p. 100-110.

3. H. E. SALZER, "Alternative formulas for osculatory and hyperosculatory inverse interpolation," Math. Comp., v. 14, 1960, p. 257-261.

4. C. Lanczos, Applied Analysis, Prentice Hall, Englewood Cliffs, New Jersey, 1956, p. 408-410, table on p. 529 . 